\title{
Cytokine Regulation of Antigen-driven Immunoglobulin Production in Filarial Parasite Infections in Humans
}

\author{
C. L. King, E. A. Ottesen, and T. B. Nutman \\ Laboratory of Parasitic Diseases, National Institutes of Health, Bethesda, Maryland 20892
}

\begin{abstract}
To define the immunoregulatory mechanisms underlying serum IgE levels found in patients with filariasis, we studied polyclonal IgE production by peripheral blood mononuclear cells (PBMC) from 15 patients with filarial infections, with a focus on the role of interleukin- 4 (IL-4) and interferon-gamma $($ IFN- $\gamma$ ) in the generation and regulation of the response. Spontaneous in vitro IgE production was elevated in 10 of the 15 patients $(836-6,464 \mathrm{pg} / \mathrm{ml}$; normals, $<500 \mathrm{pg} / \mathrm{ml})$. Addition of filarial parasite antigen to PBMC cultures significantly stimulated polyclonal IgE production in an antigen dose-dependent manner in 10 of 12 patients tested $(P<0.001)$. The essential role of $\mathrm{IL}-4$ in the generation of this response was demonstrated when simultaneous addition of anti-IL -4 completely inhibited antigen-stimulated IgE production in all 10 patients studied. An inhibitory role of endogenously produced IFN- $\boldsymbol{\gamma}$ was also indicated when the addition of anti-IFN- $\boldsymbol{\gamma}$ to the cultures significantly augmented filarial antigen-stimulated IgE production by 33-1,238\% in these same patients. Addition of $10-1,000 \mathrm{U} / \mathrm{ml}$ of recombinant human IFN- $\gamma$ to PBMC completely inhibited parasite antigen-induced IgE production.

This study demonstrates that filarial antigen-stimulated IgE production in patients with filariasis is mediated by IL-4 and down regulated by IFN- $\gamma$ and suggests that the amount of IgE produced depends on the relative quantity of $\mathrm{IL}-4$ and IFN- $\gamma$ generated by parasite-specific T cells. (J. Clin. Invest. 1990. 85:1810-1815.) IgE • interleukin-4 • interferon 8 - filariasis
\end{abstract}

\section{Introduction}

Human filarial parasites, which affect $>200$ million people worldwide, lead to persistent infections that stimulate a broad range of host immune responses. One of these responses, the production of high levels of IgE antibody, is particularly characteristic and has been implicated as being a determinant of both the resistance to infection $(1,2)$ and the pathogenesis of the various clinical manifestations. Much of the IgE produced is not parasite specific and is therefore felt to represent polyclonal activation of B cells (3). This induction of IgE by parasitic infections has been shown to be $T$ cell dependent both in animal model systems $(4,5)$ and in humans $(6)$. These $T$ cells appear to exert their effect through the production of soluble factors that selectively enhance IgE production by human B cells in vitro, as demonstrated in experiments both with parasite-specific $T$ cell clones (6) and with peripherial blood $T$ cells

Received for publication 6 November 1989 and in revised form 24 January 1990.

The Journal of Clinical Investigation, Inc.

Volume 85, June 1990, 1810-1815 from patients with severe atopy (7-9) or the hyperimmunoglobulin-E (HIE) ${ }^{1}$ syndrome (10).

In murine systems, recent experimental studies to identify the lymphokines involved in regulating the IgE response have indicated a crucial role for interleukin-4 (IL-4) in the generation of IgE and for interferon-gamma (IFN- $\gamma$ ) in suppressing this response (11). Similar studies with normal human peripheral blood mononuclear cells (PBMC) have shown that purified human recombinant IL-4 can induce IgE production in unfractionated human PBMC and that recombinant human IFN- $\gamma$ can inhibit this IL-4-stimulated IgE production (12). Furthermore, IFN- $\gamma$ has been shown to suppress the excessive ongoing IgE production, both in vitro and in vivo, in patients with the very unusual HIE syndrome (13).

In the present study, we have examined whether these mechanisms for regulating IgE production initially defined in murine systems and in patients with syndromes associated with abnormal IgE production were also involved in regulating IgE responses in the far more common situation where humans are naturally infected with helminth parasites. Using PBMC from patients with filarial infections caused by Loa loa and Onchocera volvulus, we have demonstrated in these studies that IL-4 and IFN- $\gamma$, indeed, play crucial roles in the regulation of the polyclonal IgE responses commonly seen in these infections.

\section{Methods}

Patient population. 10 patients with Loa loa and five patients with Onchocerca volvulus were studied (Table I). All were in good health at the time of study except for the signs and symptoms of their parasite infections. Onchocerciasis was confirmed in four patients by the presence of microfilariae in skin snips; the fifth patient (No. 15) had had documented infection 17 yr previously, but had subsequently cleared the infection. Loa loa infections were documented by $(a)$ microfilariae in the blood (patient Nos. 4 and 13), (b) removal of an adult worm from the skin after treatment (patient Nos. 1, 2, and 9), (c) the occurrence of a migrating worm across the eye (patient Nos. 5 and 7), or (d) satisfying the clinical criteria for loiasis (14) by having Calabar swellings (localized angioedema) and eosinophilia (patient Nos. 3, 6, and 12). 13 patients (Nos. 1-3 and 5-14) were expatriates who had acquired their infections during the course of their work in endemic areas; patient Nos. 4 and 15 were native to the endemic areas. The majority of patients (Nos. 1-3, 5-7, 12, and 13) were studied before any treatment was given; the remaining received at least one course of therapy before the time of study (either diethycarbamazine or ivermectin) that had not been effective in completely eradicating the infections.

Antigens and mitogens. Brugia malayi filarial antigen (BmA) and Onchocerca volvulus antigen (OvA) were prepared as saline extracts of

1. Abbreviations used in this paper: HIE, hyperimmunoglobulin-E; IFN- $\gamma$, interferon-gamma; IL-4, interleukin-4; PBMC, peripheral blood mononuclear cells. 
Table I. Patient Population Studied

\begin{tabular}{rlccrr}
\hline & & & & & \\
Patient & \multicolumn{1}{c}{ Disease } & Age/sex & $\begin{array}{c}\text { IgG antifilarial } \\
\text { antibody level }\end{array}$ & $\begin{array}{c}\text { Spontaneous } \\
\text { in vitro IgE } \\
\text { production }\end{array}$ \\
\hline & & & $U / m l$ & $n g / m l$ & $n g / m l$ \\
1 & Loiasis & 26/F & 39,310 & 2,005 & 4.522 \\
2 & Loiasis & $31 / \mathrm{F}$ & 1,582 & 1,326 & 2.435 \\
3 & Loiasis & $26 / \mathrm{M}$ & 5,997 & 925 & 1.312 \\
4 & Loiasis & $34 / \mathrm{M}$ & 2,097 & 14,650 & 3.267 \\
5 & Loiasis & $26 / \mathrm{M}$ & 4,331 & 1,855 & 1.649 \\
6 & Loiasis & $27 / \mathrm{F}$ & 1,965 & 9,000 & 1.389 \\
7 & Loiasis & $33 / \mathrm{F}$ & 2,644 & 483 & 0.836 \\
8 & Onchocerciasis & $37 / \mathrm{F}$ & 306 & 85 & $<0.100$ \\
9 & Loiasis & $28 / \mathrm{F}$ & 445 & $<50$ & $<0.100$ \\
10 & Onchocerciasis & $6 / \mathrm{M}$ & 3,354 & 11,590 & 6.464 \\
11 & Onchocerciasis & $39 / \mathrm{M}$ & 1,321 & 1,498 & 3.427 \\
12 & Loiasis & 26/M & 803 & 19,817 & 3.015 \\
13 & Loiasis & $26 / \mathrm{F}$ & 1,398 & 1,045 & $<0.100$ \\
14 & Onchocerciasis & $31 / \mathrm{M}$ & 769 & 69 & $<0.100$ \\
15 & Onchocerciasis & $38 / \mathrm{F}$ & 60 & 219 & 0.150 \\
& & & & & \\
\hline
\end{tabular}

adult parasites as described previously $(15,16)$. Tetanus toxoid was obtained from the Massachusetts Public Health Service (Boston, MA). Streptolysin O was obtained from Difco Co. (Detroit, MI).

Culture conditions for in vitro antibody production. PBMC were obtained from heparinized blood purified by sedimentation on a Ficoll-diatrizoate gradient. Cell cultures for the measurement of immunoglobulin (Ig) production were performed in RPMI 1640 containing $10 \%$ FCS, gentamicin $(80 \mu \mathrm{g} / \mathrm{ml})$, and $25 \mathrm{mM}$ Hepes. PBMCs were placed in flat-bottomed wells of microtiter plates at a concentration of $2 \times 10^{6}$ cells $/ \mathrm{ml}$ in C-RPMI (total volume $1 \mathrm{ml}$ ). To these cultures were added media alone, cycloheximide $(100 \mu \mathrm{g} / \mathrm{ml}$; Sigma Chemical Co. St. Louis, MO), varying concentrations of filarial antigen, and/or different concentrations of recombinant IFN- $\gamma$ (Genentech, Inc., San Francisco, CA), rabbit anti-human IL-4 (Genzyme Corp., Boston, MA), rabbit anti-human IFN- $\gamma$, or normal rabbit serum. All cultures were done in quadruplicate and incubated at $37^{\circ} \mathrm{C}$ in $5 \% \mathrm{CO}_{2}$ for 14-16 d. The amount of spontaneous in vitro Ig production is defined as the amount produced in the experimental condition after subtracting the amount produced by the identical cultures performed in parallel but containing cyclohexamide.

Repeat cultures of PBMC on the same patients in separate experiments always gave similar, if not identical, results for spontaneous in vitro IgE production and for stimulation of IgE production by filarial antigens. Spontaneous in vitro IgE production by PBMC from five normal individuals ranged from undetectable to $500 \mathrm{pg} / \mathrm{ml}$ in this assay.

ELISA assays for IgM, IgE, and parasite-specific IgG antibody. IgM in culture supernatants was measured using an enzyme-linked immunosorbent assay described previously (17). IgE was measured using an avidin-biotin amplified ELISA described previously (18). Serum parasite-specific IgG antibodies to $\mathrm{BmA}$ were measured as previously described (16).

Statistical analyses. Data are expressed as mean \pm SEM unless otherwise stated. Experimental conditions were compared using a $t$ test. Correlations of groups of ordered data were determined by Spearman's rank correlation.

\section{Results}

Serum IgE levels and spontaneous in vitro IgE production in patients with filariasis. 11 of 15 study patients had elevated serum IgE levels which ranged from 483 to $19,817 \mathrm{ng} / \mathrm{ml}$ (normals $<350 \mathrm{ng} / \mathrm{ml}$; Table I). The four patients with normal serum IgE levels (Nos. 8, 9, 14, and 15) had been previously treated for filariasis, and the only remaining evidence of active infection in three of those (Nos. 8, 9, and 14) was the persistance of elevated antifilarial antibody titers (normals $<150$ $\mathrm{U} / \mathrm{ml}$; Table I); patient No. 15 appeared to have spontaneously cured her infeetion acquired $17 \mathrm{yr}$ previously. There was a trend (not statistically significant) for patients with normal serum IgE levels to have the lowest antifilarial antibody titers.

Spontaneous IgE production by cultured PBMC from the same patients ranged from $<100 \mathrm{pg} / \mathrm{ml}$ to $6.464 \mathrm{ng} / \mathrm{ml}$. Though there was a tendency toward a correlation between levels of serum IgE and spontaneous in vitro IgE production (Table I), this relationship did not reach statistical significance.

Effect of filarial antigens on in vitro IgE production by $P B M C$. When the effect of parasite antigen on IgE production in patients with filarial infection was studied in 12 of the 15 patients, 10 of 12 showed a significant increase in the in vitro IgE production when PBMC were stimulated with BmA (for patients with loiasis) or OvA (for patients with onchocerciasis). Among the six patients with loiasis demonstrating a significant increase in IgE production, five showed a dose-dependent response that peaked at $0.001 \mu \mathrm{g} / \mathrm{ml}$ of BmA and was suppressed at higher concentrations (Fig. 1). In the sixth patient (No. 12), IgE production was stimulated by doses of $\mathrm{BmA}$ as low as $0.001 \mu \mathrm{g} / \mathrm{ml}$, but peak response was achieved only at a concentration of $1 \mu \mathrm{g} / \mathrm{ml}$ of BmA (data not shown). Among the four patients with onchocerciasis who demonstrated a significant increase in IgE production after stimulation by OvA, all showed dose-dependent responses to OvA, but the peak response of in vitro IgE production to parasite antigen differed among the individual patients. Patient No. 8 had peak IgE responses at $0.01 \mu \mathrm{g} / \mathrm{ml} \mathrm{OvA}$, patient No. 15 at $0.1 \mu \mathrm{g} / \mathrm{ml}$, and patient Nos. 10 and 11 at $1 \mu \mathrm{g} / \mathrm{ml}$ OvA.

In these 10 patients, who responded to parasite antigen, peak in vitro IgE production in response to parasite antigens correlated significantly with net spontaneous in vitro IgE synthesis $\left(r_{\mathrm{S}}=0.90, P<0.001\right)$. Furthermore, the two patients whose PBMC did not respond to filarial antigens in vitro actually had normal serum IgE levels and undetectable levels of spontaneous in vitro IgE production. There was no correlation between patients' in vitro IgE responses to parasite antigens and their serum antifilarial IgG levels.

The addition of varying concentrations of nonparasite antigens (SLO and tetanus toxiod) to PBMC in four patients

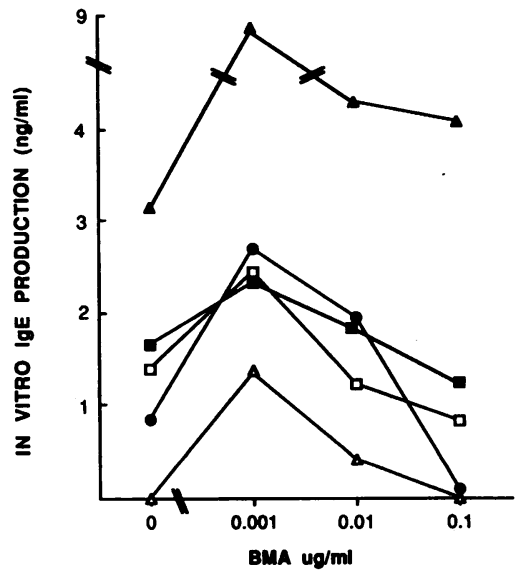

Figure 1. Effect of addition of different concentrations of $\mathrm{BmA}$ on IgE production by PBMC from five patients with loiasis. Each point represents the mean of quadruplicate cultures. Patient Nos. 4 , $5,6,7$, and 9 are represented by a solid triangle, solid square, open square, solid circle, and open triangle, respectively. 


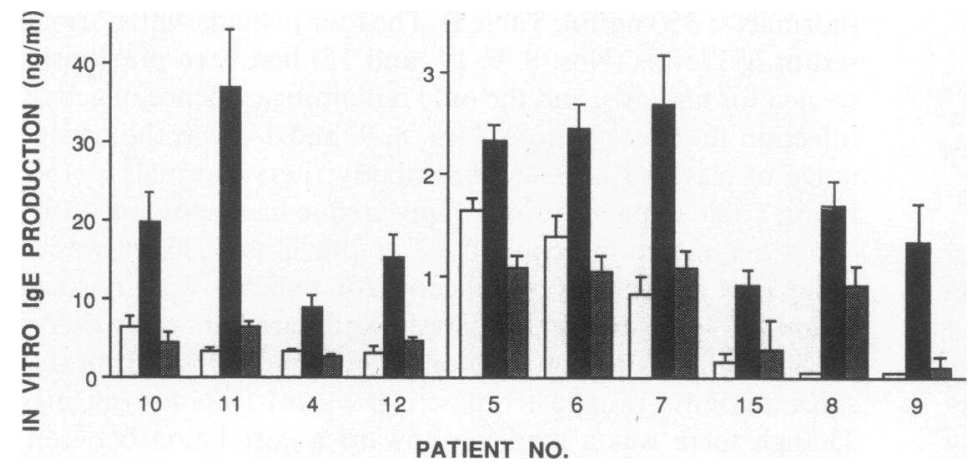

Figure 2. Effect of anti-IL-4 on parasite antigen-induced IgE production. The ordinate indicates the amount of IgE produced in culture, and for patient Nos. 10, 11, 4, and 12 the scale ranges from 0 to $40 \mathrm{ng} / \mathrm{ml}$, whereas the scale for the other patients ranges from 0 to $3 \mathrm{ng} / \mathrm{ml}$. Each bar represents the mean \pm SEM IgE production of quadruplicate cultures in the presence of media alone (open bars) or after the addition of parasite antigen (dark bars), or parasite antigen + anti-IL-4 (shaded bars). Patients are presented in rank order of spontaneous in vitro IgE production.

(Nos. 7, 12, 14, and 15) had no effect on IgE production (data not shown), and the addition of BmA (three experiments) or OvA (one experiment) to PBMC cultures from normal individuals failed to induce any IgE production in vitro (data not shown).

Effect of anti-IL-4 on parasite antigen-induced IgE production. To examine the role of IL-4 on antigen-driven IgE production, PBMC were cultured in the presence of both parasite antigen and $1 \mu \mathrm{g} / \mathrm{ml}$ of anti-IL-4. In 9 of the 10 patients responsive to parasite antigen, the antigen-induced IgE production (studied at each individual's peak response) was completely inhibited by anti-IL-4, and in the remaining patient, No. 8 , it was significantly inhibited (by $46 \% ; P<0.05$; Fig. 2 ). This effect was dose-dependent in that decreasing the concentration of anti-IL-4 in the presence of parasite antigen reduced the inhibition of parasite antigen-induced IgE production (data not shown). Addition of normal rabbit sera to parallel cultures at the same dilution as the anti-IL-4 antiserum had no effect on the antigen-induced IgE response.

Effect of anti-IFN- $\gamma$ on parasite antigen-induced IgE production. The role of IFN- $\gamma$ in the regulation of antigen-induced IgE responses was studied by culturing PBMC in the presence of both parasite antigen and rabbit anti-IFN- $\gamma$. In all 10 parasite antigen-responsive patients, the presence of antiIFN- $\gamma$ significantly increased IgE production by $33 \%$ to $1,238 \%$ (Fig. 3). Control cultures containing normal rabbit

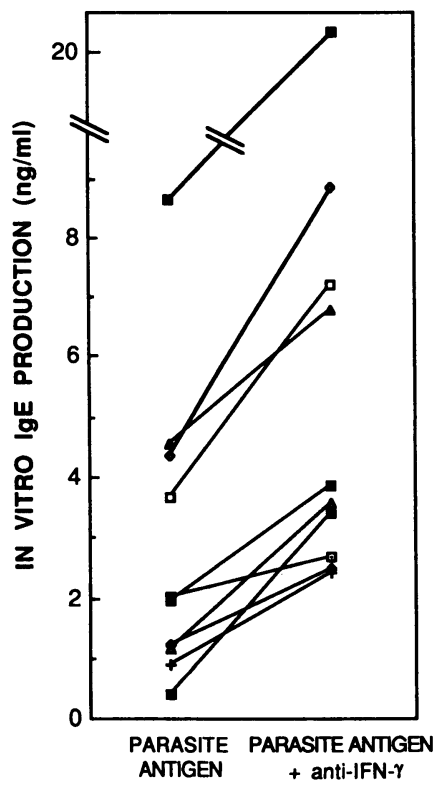

Figure 3. Peak effect of antiIFN- $\gamma$ on IgE production by PBMC cultures containing parasite antigen in the presence or absence of anti-IFN- $\gamma$. Each line represents an individual patient. sera (at the same concentration) had no effect on parasite antigen induced IgE production.

Augmentation of parasite antigen-induced in vitro IgE production by anti-IFN- $\gamma$ was dependent on antigen concentration. Addition of anti-IFN- $\gamma$ to PBMC cultures containing concentrations of parasite antigen that induced maximal IgE production did not increase further the IgE production in 9 of 10 antigen responsive patients (data not shown); rather, maximal anti-IFN- $\gamma$ augmentation of parasite antigen-induced IgE production occurred at those higher antigen concentrations that actually suppressed IgE production (Fig. 1). This relationship between antigen concentration and the effect of antiIFN- $\gamma$ on IgE production is illustrated in Fig. 4, where data from the five loiasis patients whose antigen-induced IgE production had similar dose-response curves (Fig. 1) are presented. Anti-IFN- $\gamma$ was unable to augment in vitro IgE production stimulated by $0.001 \mu \mathrm{g} / \mathrm{ml}$ of $\mathrm{BmA}$, but in the presence of antigen levels that suppressed IgE production ( 0.01 and $0.1 \mu \mathrm{g} / \mathrm{ml}$ ), anti-IFN- $\gamma$ actually increased in vitro IgE production back to maximal levels (Fig. 4). In contrast, addition of anti-IFN- $\gamma$ to PBMC cultures without parasite antigen had no effect on spontaneous in vitro IgE production in five of six parasite patients so studied; and in the sixth patient (No. 8), addition of anti-IFN- $\gamma$ increased IgE production from undetectable to only minimal levels.

Effect of IFN- $\gamma$ on spontaneous and parasite antigen-induced IgE production by PBMC. To investigate further the effect of IFN- $\gamma$ on in vitro IgE production, separate experiments were performed in which IFN- $\gamma$ was added to PBMC cultures containing optimal parasite antigen concentrations

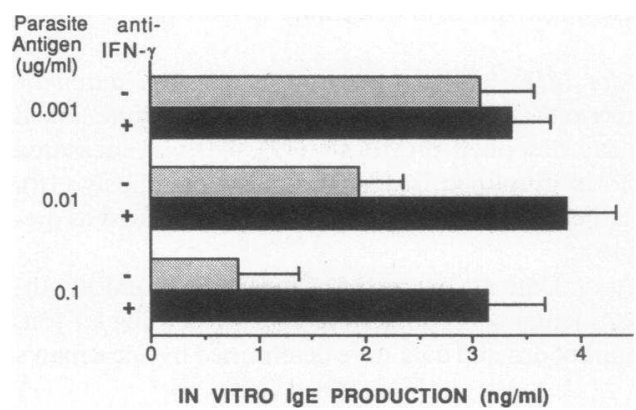

Figure 4. Effect of anti-IFN- $\gamma$ on the amount of IgE induced by different concentrations of parasite antigen. Each bar represents the mean \pm SEM IgE production of the same five patients with loiasis, whose dose-response curves are depicted in Fig. 1. Shaded bars represent cultures with parasite antigen alone, and dark bars represent paired cultures with both parasite antigen and anti-IFN- $\gamma$. 


\begin{tabular}{|c|c|c|c|c|c|}
\hline \multirow[b]{2}{*}{$\mathrm{BmA}$} & \multirow[b]{2}{*}{ IFN- $\boldsymbol{\gamma}$} & \multicolumn{4}{|c|}{ IgE production } \\
\hline & & PT 4 & PT 6 & PT 7 & PT 5 \\
\hline $0.001 \mu \mathrm{g} / \mathrm{ml}$ & $U / m l$ & $n g / m l$ & $n g / m l$ & $n g / m l$ & $n g / m l$ \\
\hline- & 0 & $3.267 \pm 0.33$ & $1.556 \pm 0.27$ & $0.902 \pm 0.13$ & $1.649 \pm 0.09$ \\
\hline+ & 0 & $8.982 \pm 1.8$ & $4.029 \pm 0.56$ & $3.401 \pm 0.31$ & $2.323 \pm 0.16$ \\
\hline+ & 10 & $2.094 \pm 0.30(>100)$ & $2.988 \pm 0.28(42)$ & $1.888 \pm 0.12(61)$ & $1.507 \pm 0.05(>100)$ \\
\hline+ & 1,000 & $1.856 \pm 0.06(>100)$ & $1.438 \pm 0.42(>100)$ & $1.408 \pm 0.22(80)$ & $1.266 \pm 0.04(>100)$ \\
\hline+ & 100,000 & $1.107 \pm 0.27(>100)$ & $0.646 \pm 0.31(>100)$ & $1.042 \pm 0.07(94)$ & $1.176 \pm 0.22(>100)$ \\
\hline
\end{tabular}

Values in parentheses indicate percent inhibition of the $\mathrm{BmA}$ antigen-stimulated IgE production, defined as:

$$
(1-[\operatorname{IgE} \text { in the presence of IFN- } \gamma-\text { spontaneous IgE }] /[\operatorname{IgE} \text { induced by antigen }- \text { spontaneous IgE }]) \times 100
$$

for four patients with loiasis (Table II). Addition of 10-1,000 $\mathrm{U} / \mathrm{ml}$ of IFN- $\gamma$ to cultures completely or almost completely inhibited parasite antigen induced IgE production in all four patients. Concentrations of $1,000-100,000 \mathrm{U} / \mathrm{ml}$ of IFN- $\gamma$ further inhibited in vitro IgE production to levels significantly below that spontaneously released from PBMC cultured in the absence of parasite antigen for three of the four patients.

To determine whether IFN- $\gamma$ could inhibit spontaneous in vitro IgE production in PBMC cultures by itself, separate studies were performed. When IFN- $\gamma$ was added to unstimulated PBMC cultures of five patients with loiasis, concentrations of IFN- $\gamma$ as low as $10 \mathrm{U} / \mathrm{ml}$ significantly inhibited $(P<0.001)$ IgE production by an average of $35 \%$ (Fig. $5,-\bullet$ ). Inhibition increased with increasing doses, reaching $82 \%$ inhibition at $10^{5} \mathrm{U} / \mathrm{ml}$ IFN- $\gamma$. In contrast, in vitro IgM production was not significantly inhibited by 10 to $1000 \mathrm{U} / \mathrm{ml}$ of IFN- $\gamma$, and at $10^{5} \mathrm{U} / \mathrm{ml} \mathrm{IFN}-\gamma$ IgM production was inhibited by only 16\% (Fig. 5, O-O).

\section{Discussion}

The current understanding of cytokine regulation of IgE production in humans is based largely on results of experiments in which recombinant lymphokines were added to cultured lymphocytes from normal or atopic individuals. Whether such findings also pertain to the in vivo generation of IgE in disorders associated with elevated serum levels of IgE is not as

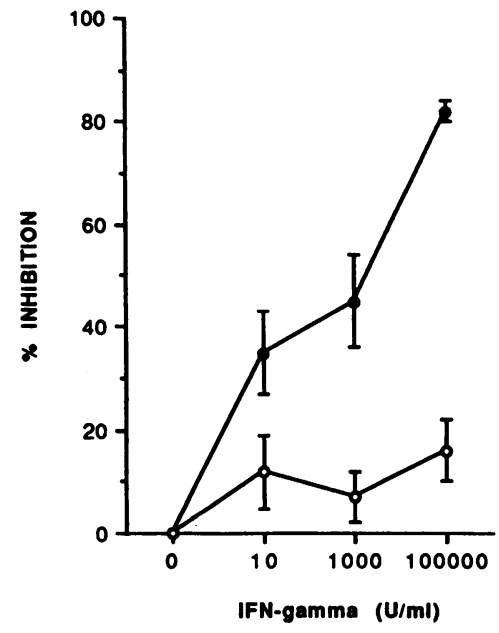

Figure 5. Effect of addition of different concentrations of IFN- $\gamma$ on spontaneous in vitro IgE (solid circles) or IgM (open circles) production by PBMC from five patients with loiasis. The data are expressed as percent inhibition ( \pm SEM) of Ig produced in the absence of added IFN- $\gamma$. well-understood, partly because an adequate in vitro model of antigen-induced B lymphocyte production of IgE has not been previously available. The present study extends the development of such a model (18) and demonstrates that IL-4 and IFN- $\gamma$ are important mediators for the regulation of parasite antigen-stimulated IgE production in humans.

$B$ cell activation and immunoregulation were initially analyzed using in vitro systems of antigen-induced antibody production by cells obtained from subjects immunized with either recall (19) or virgin (20) antigens. Such investigations were later extended to naturally acquired filarial infections by using parasite antigens to induce both total and parasite antigen-specific IgG and IgM production (16). The present study, using a similar in vitro system, has demonstrated that parasite antigen can also induce polyclonal IgE production at concentrations that are similar to those required for IgG and IgM production (16) and that are several orders of magnitude lower than those optimal for antigen-induced lymphocyte proliferation. Such parasite antigen-stimulated polyclonal IgE production was clearly dose dependent, but while the antigen concentrations needed to induce optimal IgE by PBMC were similar for the majority of filariasis patients $(0.01-0.001 \mu \mathrm{g} / \mathrm{ml})$, peak IgE production occurred at significantly higher parasite antigen concentrations in four others. Such differences may reflect only variable responses of each individual's $\mathrm{T}$ lymphocytes to allergens found at different concentrations in the complex preparations of parasite antigen used in this study, and efforts are underway to study the effect of defined/purified (21) or cloned filarial antigens (22) on T and B cell responses.

An interesting observation made in this study was that higher concentrations of parasite antigen suppressed antigeninduced IgE production and that this suppression was reversible with the simultaneous addition of anti-IFN- $\gamma$. This finding suggests that concentrations of parasite antigen that induce optimal IgE production may selectively stimulate antigen-specific $T$ cells to produce primarily IL-4. Higher concentrations of parasite antigen may stimulate proportionally more IFN- $\gamma$ production by either the same parasite antigen-specific $\mathrm{T}$ cells, by additional $\mathrm{CD}^{+}$cells that produce predominantly IFN- $\gamma$, or by $\mathrm{T}$ suppressor cells. These results are consistent with those of previous studies of other immunoglobulin isotypes demonstrating that higher concentrations of antigen can suppress immunoglobulin production and that this inhibition is, in part, mediated by $\mathrm{T}$ suppressor cells (23); those earlier reports did not define the lymphokines mediating such suppression. 
While this study has demonstrated an important role for IFN- $\gamma$ in modulating the IgE response to parasite antigens, the role of IFN- $\alpha$ and prostaglandin $\mathrm{E}_{2}$ (shown previously to inhibit IL-4-induced IgE production in normal individuals [12]) in modulating IgE responses to parasite antigens remains to be defined.

This study also demonstrates that parasite antigen-induced in vitro IgE production by PBMC from patients with filariasis is IL-4 dependent. As such, they support similar observations of Nippostrongylus brasiliensis-induced IgE production in infected mice, where in vivo injection of anti-IL-4 completely blocked the IgE response to the parasite (24). They also support and extend the findings from a recent study in humans in which only the Dermatophagoides spp. (house dust mite)-specific $\mathrm{T}$ cell clones that expressed $\mathrm{mRNA}$ for IL-4 were capable of inducing IgE production by autologous B cells (25); however, even that study failed to demonstrate directly that endogenously produced IL-4 from the antigen-specific clones, in fact, induced the IgE production.

In murine systems, IL-4 may have a role not just in initiating but also in maintaining an established ongoing in vivo IgE response (26). Whether IL-4 has such a role in humans is less clear. Addition of anti-IL-4 to unstimulated cultures of PBMC from patients with filariasis (reported in this study) and from HIE patients (unpublished data) failed to inhibit the excessive ongoing IgE production. Whether this finding is a technical artifact resulting from culture conditions, or whether it indicates that the primary role of IL-4 in humans may be to induce isotype switching from IgM to IgE and not in sustaining the IgE response, remains to be determined. There is evidence that another cytokine, macrophage-derived IL-6, may be important in both the IL-4-dependent IgE induction (27) and in sustaining ongoing immunoglobulin production by acting as a non-isotype-specific, late amplification signal for Ig synthesis by B cells (28).

Our study further indicates that rècombinant human IFN- $\gamma$, at concentrations of $10-100,000 \mathrm{U} / \mathrm{ml}$, can essentially completely inhibit parasite antigen-induced IgE production in vitro (Table II). This finding is similar to results seen in murine systems in which in vivo IgE production was inhibited by simultaneous administration of IFN- $\gamma$ and augmented by injection of anti-IFN- $\gamma$ antibody (24). Additionally, the data also show that IFN- $\gamma$ can inhibit the ongoing spontaneous production of $\mathrm{IgE}$ in cultures of PBMC from patients with filariasis, whether by inhibiting IgE secreting plasma cells or by inhibiting the IL-4-induced IgE production is uncertain but the latter possibility is less likely for several reasons. First, as mentioned above, spontaneous in vitro IgE production by cultured PBMC from HIE or filariasis patients appears not to be dependent on the ongoing production of IL-4. Second, T cells require a stimulus for cytokine production which is absent in these experiments, except for the presence of IFN- $\gamma$. It remains to be determined whether IFN- $\gamma$ exerts its inhibitory effect directly on plasma cells or indirectly through $\mathrm{T}$ cells or macrophages.

The present study thus provides a model of antigen-stimulated IgE production that enables further dissection of the network of cellular components and cytokines involved in the regulation of IgE antibody production in humans. This model can be used to assess not only polyclonal IgE production, but also regulation of antigen-specific antibody production; in addition, it should allow one to determine whether IgG subclass production (both total and antigen specific) is also under the influence of regulatory controls similar to those of IgE, as suggested in studies on animal models (29). Indeed, because elevated serum IgE levels in patients with lymphatic filariasis generally correlate with high serum $\operatorname{IgG}_{4}(30)$, there is a suggestion that parallel immunoregulatory mechanisms may be at work. Examination of the frequency of parasite-specific $T$ and B lymphocytes, differences in the in vitro responses to parasite antigen by PBMC, and the role of different cytokines in the generation of the IgE response among such patients with filarial parasite infections should provide a greater understanding of the immunoregulatory network underlying not only these, but also other disorders associated with elevated levels of serum IgE.

\section{References}

1. Ottesen, E. A. 1980. Immunopathology of lymphatic filariasis in man. Springer Semin. Immunopathol. 2:373-385.

2. Dessein, A. J., W. L. Parker, S. L. James, and J. R. David. 1981. IgE antibody and resistance to infection. I. Selective suppression of the IgE antibody response in rats diminishes the resistance and the eosinophil response to Trichinella spiralis infection. J. Exp. Med. 153:423436.

3. Hussain, R., R. G. Hamilton, V. Kumaraswami, N. F. Adkinson, and E. A. Ottesen. 1981. IgE response in human filariasis. I. Quantitation of filaria-specific IgE. J. Immunol. 127:1623-1629.

4. Okumura, K., and T. Tada. 1971. Regulation of homocytotropic antibody formation in the rat. III. Effect of thymectomy and splenectomy. J. Immunol. 106:1019-1025.

5. Tada, T., M. Taniguchi, and K. Okumura. 1971. Regulation of homocytotropic antibody formation in the rat. II. Effect of $\mathrm{X}$ irradiation. J. Immunol. 106:1012-1018.

6. Nutman, T. B., D. J. Volkman, R. Hussain, A. S. Fauci, and E. A. Ottesen. 1985. Filarial parasite-specific T cell line: induction of IgE synthesis. J. Immunol. 134:1178-1184.

7. Saxon, A., C. Morrow, and R. H. Stevens. 1980. Subpopulations of circulating B cells and regulatory $\mathrm{T}$ cells involved in in vitro immunoglobulin E production in atopic patients with elevated serum immunoglobulin E. J. Clin. Invest. 65:1457-1468.

8. Romagnani, S., E. Maggi, G. F. Del Prete, and M. Ricci. 1983. IgE synthesis in vitro induced by $T$ cell factors from patients with elevated serum IgE levels. Clin. Exp. Immunol. 52:85-88.

9. Umetsu, D. T., D. Y. M. Leung, R. Siraganian, H. H. Jabara, and R. S. Geha. 1985. Differential requirements of $B$ cells from normal and allergic subjects for the induction of IgE synthesis by an alloreactive T cell clone. J. Exp. Med. 162:202-214.

10. Saryan, J. A., D. Y. Leung, and R. S. Geha. 1983. Induction human IgE synthesis by a factor derived from $\mathrm{T}$ cells of patients with hyper-IgE states. J. Immunol. 130:242-247.

11. Snapper, C. M., and W. E. Paul. 1987. Interferon-gamma and B cell stimulatory factor-1 reciprocally regulate Ig isotype production. Science (Wash. DC). 236:944-947.

12. Pene, J., F. Rousset, F. Briere, I. Chretten, J. F. Bonnefoy, H. Spits, T. Yokota, N. Arai, K. Arai, J. Banchereau, and J. E. deVries. 1988. IgE production by normal human lymphocytes is induced by interleukin 4 and suppressed by interferons $\alpha$ and $\gamma$ and prostaglandin E 2 . Proc. Natl. Acad. Sci. USA. 85:6680-6684.

13. King, C. L., J. I. Gallin, H. L. Malech, S. L. Abramson, and T. B. Nutman. 1989. Regulation of immunoglobulin production in hyperimmunoglobulin-E recurrent-infection syndrome by IFN- $\gamma$. Proc. Natl. Acad. Sci. USA. 86:10085-10089.

14. Nutman, T. B., K. D. Miller, M. Mulligan, and E. A. Ottesen. 1986. Loa loa infection in temporary residents of endemic regions: recognition of a hyperresponsive syndrome with characteristic clinical manifestations. J. Infect. Dis. 154:1-10. 
15. Hamilton, R. G., R. Hussein, E. A. Ottesen, and N. F. Adkinson. 1981. The quantitation of parasite-specific human IgG and IgE in sera: evaluation of solid phase RIA and ELISA methodology. J. Immunol. Methods. 44:101-114.

16. Nutman, T. B., A. S. Withers, and E. A. Ottesen. 1985. In vitro parasite antigen-induced antibody responses in human helminth infections. J. Immunol. 135:2794-2799.

17. Nutman, T. B., V. Kumaraswami, L. Pao, P. R. Narayanan, and $E$. A. Ottesen. 1987. An analysis of in vitro $B$ cell immune responsiveness in human lymphatic filariasis. J. Immunol. 138:3954-3959.

18. Nutman, T. B., R. Hussain, and E. A. Ottesen. 1984. IgE production in vitro by peripheral blood mononuclear cells of patients with parasitic helminth infections. Clin. Exp. Immunol. 58:174-182.

19. Volkman, D. J., S. P. Allyn, and A. S. Fauci. 1982. Antigen-induced in vitro antibody production in humans: tetanus toxoid-specific antibody synthesis. J. Immunol. 129:107.

20. Lane, H. C., D. J. Volkman, G. Whealen, and A. S. Fauci. 1981. In vitro antigen-induced antigen-specific antibody production in man. J. Exp. Med. 154:1043.

21. Lal, R. B., T. B. Lynch, and T. B. Nutman. 1987. Brugia malayi antigens associated with lymphocyte activation in filariasis. $J$. Immunol. 139:1652-1657.

22. Unnasch, T. R., M. Y. Gallin, P. T. Soboslay, K. D. Erttmann, and B. M. Greene. 1988. Isolation and characterization of expression cDNA clones encoding antigens of Onchercerca volvulus infective larvae. J. Clin. Invest. 82:262-269.

23. Lamb, J. R., B. J. Skidmore, N. Green, J. M. Chiller, and M. Feldmann. 1983. Induction of tolerance in influenza virus: immune $T$ lymphocyte clones with synthetic peptides of influenza hemagglutinin. J. Exp. Med. 157:1434-1447.
24. Finkelman, F. D., I. M. Katona, T. R. Mosmann, and R. L. Coffman. 1988. Interferon- $\gamma$ regulates the isotypes of immunoglobulin secreted during in vivo humoral immune response. J. Immunol. 140:1022-1027.

25. O'Hehir, R. E., V. Bal, D. Quint, R. Moqbel, A. B. Kay, E. D. Zanders, and J. R. Lamb. 1989. An in vitro model of allergen-dependent IgE synthesis by human B lymphocytes: comparison of the response of an atopic and non-atopic individual to Dermatophagoides spp. (house dust mite). Immunology. 66:499-504.

26. Finkelman, F. D., I. M. Katona, J. F. Urban, J. Holmes, J. Ohara, A. S. Tung, J. vG. Sample, and W. E. Paul. 1988. Interleukin-4 is required to generate and sustain in vivo IgE responses. J. Immunol. 141:2335-2341.

27. Vercelli, D., H. H. Jabara, K. Arai, T. Yokota, and R. S. Geha. 1989. Endogenous interleukin 6 plays an obligatory role in interleukin 4-dependent human IgE synthesis. Eur. J. Immunol. 19:1419-1424.

28. Muraguchi, A., T. Hirano, B. Tang, T. Matsudea, Y. Horii, K. Nakajima, and T. Kishimoto. 1988. The essential role of B cell stimulatory factor 2 (BSF-2/IL-6) for the terminal differentiation of B cells. J. Exp. Med. 167:332-344.

29. Coffman, R. L., B. W. Seymour, D. A. Lebman, D. D. Hiraki, J. A. Christiansen, B. Shrader, H. M. Cherwinski, H. F. Savelkoul, F. D. Finkelman, and M. W. Bond. 1988. The role of helper T cell products in mouse B cell differentiation and isotype regulation. Immunol. Rev. 102:5-28.

30. Ottesen, E. A., F. Skvaril, S. P. Tripathy, R. W. Poindexter, and R. Hussein. 1985. IgG subclasses in human filariasis: prominence of IgG4 in the IgG antibody response to human filariasis. J. Immunol. 134:2702-2712. 